\title{
Tunability of solitary wave properties in one-dimensional strongly nonlinear phononic crystals
}

\author{
C. Daraio, ${ }^{1}$ V. F. Nesterenko, ${ }^{1,2, *}$ E. B. Herbold, ${ }^{2}$ and S. Jin ${ }^{1,2}$ \\ ${ }^{1}$ Materials Science and Engineering Program, University of California at San Diego, La Jolla, California 92093-0418, USA \\ ${ }^{2}$ Department of Mechanical and Aerospace Engineering, University of California at San Diego, La Jolla, California 92093-0411, USA
}

(Received 16 June 2005; published 16 February 2006)

\begin{abstract}
One-dimensional strongly nonlinear phononic crystals were assembled from chains of PTFE (polytetrafluoroethylene) and stainless-steel spheres with gauges installed inside the beads. Trains of strongly nonlinear solitary waves were excited by impacts. A significant modification of the signal shape and an increase of solitary wave speed up to two times (at the same magnitude of dynamic contact force) were achieved through a noncontact magnetically induced precompression of the chains. The data for the PTFE based chains are presented for the first time and the data for the stainless-steel beads chains are extended into a range of maximum dynamic forces more than one order of magnitude lower than previously reported. Experimental results agreed reasonably well with the long-wave approximation and numerical calculations based on the Hertz interaction law for particles interactions.
\end{abstract}

DOI: 10.1103/PhysRevE.73.026610

PACS number(s): 05.45.Yv, 45.70.-n, 43.25.+y, 46.40.Cd

\section{INTRODUCTION}

One-dimensional (1D) strongly nonlinear chains of beads are presently a very active area of research because they provide a natural step from weakly nonlinear to strongly nonlinear wave dynamics [1-40]. Their nonlinearity stems from the Hertzian contact interaction governing the forces between the beads. These systems permit the unique possibility of tuning the wave propagation behavior from linear to weakly nonlinear and further to strongly nonlinear regimes [19].

Through simple noncontact magnetically induced static compression it is possible to tune the wave propagation response of the system from the strongly nonlinear to the weakly nonlinear regime. This method allows a fine control of the propagating signal shape and speed with an adjustable static compressive force. Novel applications in different areas may arise from understanding the basic physics of these tunable strongly nonlinear 1D systems, especially at a low magnitude range of stresses corresponding to signals used in ultrasonic diagnostics or in the audible range. For example, tunable sound focusing devices (acoustic lenses), tunable acoustic impedance materials, sound absorption layers, and sound scramblers are among the most promising engineering applications [37].

The nonclassical, strongly nonlinear wave behavior appears in the chain if the system is "weakly" compressed with a force $F_{0}[1,2,19]$. The term "weakly" is used when the static compressive force $\left(F_{0}\right)$ is very small with respect to the maximum force in the wave. A supersonic solitary wave that propagates with a speed $V_{s}$ with a force magnitude much higher than $F_{0}$ can be closely approximated by one single positive pulse of the periodic solution corresponding to a zero static force (or $\xi_{0}=0$ ). This exact solution has a finite length equal to only five particle diameters for a Hertzian contact interaction $[1,17,19]$. In the continuum approxima-

*Electronic address: vnesterenko@ucsd.edu tion the solitary wave speed $V_{s}$ has a nonlinear dependence on the maximum strain $\xi_{m}$, which translates to a nonlinear dependence on the maximum force $F_{m}$ on the contacts between the particles in discrete chains. The speed of a solitary wave $V_{s}$ has a nonlinear dependence on the normalized maximum strain $\xi_{r}=\xi_{m} / \xi_{0}$ in the continuum approximation or, equivalently, on the normalized force $f_{r}=F_{m} / F_{0}$ in the discrete chain with beads of diameter $a$, bulk density $\rho$, Poisson's ratio $\nu$, and Young's modulus $E$ [Eq. (1), [19,37]]:

$$
\begin{aligned}
V_{s}= & c_{0} \frac{1}{\left(\xi_{r}-1\right)} \\
& \times\left\{\frac{4}{15}\left[3+2 \xi_{r}^{5 / 2}-5 \xi_{r}\right]\right\}^{1 / 2} \\
= & 0.9314\left(\frac{4 E^{2} F_{0}}{a^{2} \rho^{3}\left(1-\nu^{2}\right)^{2}}\right)^{1 / 6} \frac{1}{\left(f_{r}^{2 / 3}-1\right)}\left\{\frac { 4 } { 1 5 } \left[3+2 f_{r}^{5 / 3}\right.\right. \\
& \left.\left.-5 f_{r}^{2 / 3}\right]\right\}^{1 / 2} .
\end{aligned}
$$

The sound speed $c_{0}$ in a chain initially compressed with a force $F_{0}$ can be deduced from Eq. (1) in the limit of $f_{r}=1$ :

$$
c_{0}=\left(\frac{3}{2}\right)^{1 / 2} c \xi_{0}^{1 / 4}=0.9314\left(\frac{2 E}{a \rho^{3 / 2}\left(1-\nu^{2}\right)}\right)^{1 / 3} F_{0}^{1 / 6},
$$

where the constant $c$ is

$$
c=\sqrt{\frac{2 E}{\pi \rho\left(1-\nu^{2}\right)}} .
$$

Equation (1) also allows the calculation of the speed of weakly nonlinear solitary wave $\left(f_{\mathrm{r}}\right.$ is slightly larger than 1$)$, which is the solution of the Korteweg-de Vries equation [19].

When $f_{\mathrm{r}}$ or $\xi_{r}$ are very large [i.e., $F_{0}\left(\xi_{0}\right)$ is approaching $0]$, Eq. (1) reduces to Eq. (4) for the solitary wave speed $V_{s}$ in a "sonic vacuum" $[3,6]$ : 


$$
V_{s}=\frac{2}{\sqrt{5}} c \xi_{m}^{1 / 4}=0.6802\left(\frac{2 E}{a \rho^{3 / 2}\left(1-\nu^{2}\right)}\right)^{1 / 3} F_{m}^{1 / 6} .
$$

The term "sonic vacuum" is used for strongly nonlinear systems without a linear part in the force displacement relation between particles in which no characteristic sound speed is present $[4,10,19]$. For simplicity only the leading approximation was used to connect strains in the continuum limit and forces in discrete chain in Eqs. (1) and (4). The similarity between Eqs. (2) and (4) is striking (and can be misleading) because these equations describe qualitatively different types of wave disturbances. Equation (2) represents the speed of a sound wave with an infinitely small dynamic force magnitude in comparison with the initial precompressive force and with a long wavelength. This wave is the solution of the classical linear d'Alambert wave equation. Equation (4) corresponds to the speed of a strongly nonlinear solitary wave with a finite width of five particle diameters and an inifinite ratio of solitary wave dynamic force magnitude to initial compression. This solitary wave is the solution of the strongly nonlinear wave equation first derived in [1]. A strongly nonlinear compression solitary wave exists for any general power-law interaction between particles with an exponent in the force dependence on displacement $n>1$ (Hertz' law is a special case when $n=3 / 2$ ) $[3,5,10,18,19]$. The exponent $n$ determines the width of the solitary wave and the dependence of its speed on the maximum strain. The corresponding equations for the speed and width of these solitary waves in the continuum approximation were first derived in [3]. The results of numerical calculations for discrete chains can be found in [31]. A chain with particles interacting according to the general strongly nonlinear law also supports the strongly nonlinear compression or rarefaction solitary waves depending on an elastic "hardening" or "softening" behavior $[10,19]$.

It is possible to find from Eqs. (1)-(4) that the speed $V_{s}$ of a solitary wave propagating in a one-dimensional granular chain can be significantly smaller than the sound speed in the material composing the beads and can be considered approximately constant in any narrow interval of its relative magnitude $f_{\mathrm{r}}$ due to the small exponent in the power-law dependence. The signal speed of strongly nonlinear and linear compression waves in this condensed "soft" matter can be below the level of sound speed in gases at normal conditions as was experimentally demonstrated for PTFE- (polytetrafluoroethylene) based sonic vacuum [37]. The described properties of strongly nonlinear waves may permit the use of these materials as effective delay lines with an exceptionally low speed of the signal.

In stainless-steel-based chains, previous experimental and numerical data regarding the dependence of the solitary wave speed on the force magnitude exist for only relatively large magnitudes of dynamic forces $(20-1200 \mathrm{~N})$ [11,15] and $2-100 \mathrm{~N} \mathrm{[36]} \mathrm{and} \mathrm{for} \mathrm{large} \mathrm{diameters} \mathrm{of} \mathrm{stainless-steel}$ beads: $8 \mathrm{~mm}$ and $26 \mathrm{~mm}$, respectively. To characterize the behavior of stainless-steel-based strongly nonlinear systems at force magnitudes closer to the magnitude of the signals used in ultrasonic diagnostics we extended the range of experimental data to a lower force magnitude range down to $0.1 \mathrm{~N}$ in a chain of beads with a smaller diameter of
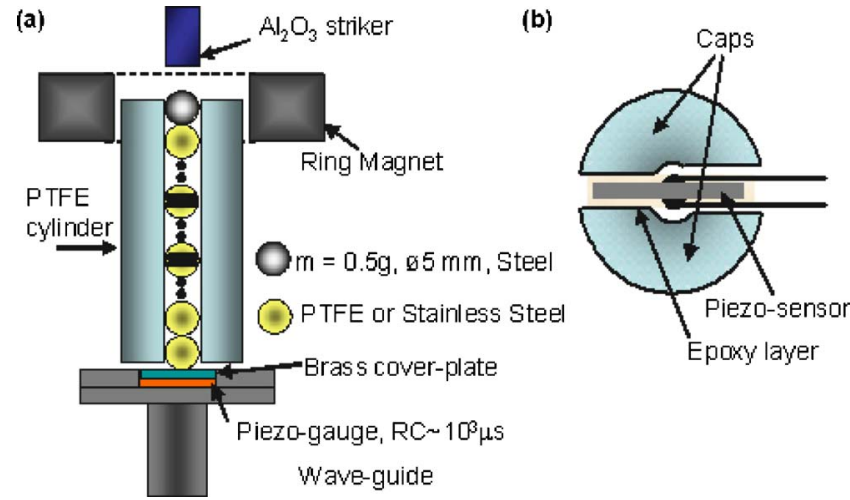

FIG. 1. (Color online) (a) Experimental setup for testing of strongly nonlinear 1D chains, two particles with embedded piezoelements are shown in the middle of the chain, the magnetic particle on the top is used for the tuning of the system behavior through exerting a compressive force on the chain equal to the mass of the magnet. The smaller black circles identify a continuation of the similar particles. (b) Schematic drawing of a particle with embedded piezosensor.

$4.76 \mathrm{~mm}$. Scaling down of the size of the beads is important for the assembling of practical devices.

\section{EXPERIMENTAL SETUP}

The speed, duration, and dynamic force magnitude in propagating and reflected pulses from the wall were measured using the experimental setup presented in Fig. 1. Onedimensional PTFE-based phononic crystals were assembled in a PTFE cylinder, with inner diameter $5 \mathrm{~mm}$ and outer diameter $10 \mathrm{~mm}$. The cylinder was filled vertically with chains of 20 and 52 PTFE balls (McMaster-Carr catalog) with diameter $a=4.76 \mathrm{~mm}$ and mass $0.1226 \mathrm{~g}$ (standard deviation $0.0008 \mathrm{~g}$ ) [Fig. 1(a)]. PTFE has a high strain rate sensitivity [41] and an exceptionally low elastic modulus in comparison with metals $[42,43]$, which ensures a very low speed of soliton propagation [Eqs. (1) and (4)]. To compare the properties of the system (signal speed, tunability) 20 stainless-steel beads (316 steel, McMaster-Carr catalog) with diameter $a=4.76 \mathrm{~mm}$ and mass $0.4501 \mathrm{~g}$ (standard deviation $0.0008 \mathrm{~g}$ ) were assembled in the same PTFE cylinder.

A magnetic steel ball, with diameter $a=5.0 \mathrm{~mm}$ and mass $0.5115 \pm 0.0001 \mathrm{~g}$, was then placed on top of the PTFE or stainless-steel chain to magnetically induce a precompression equal to the weight of the magnet [Fig. 1(a)]. This experimental setup allows the tunability of interfacial properties, as, for example, in laminar composite systems made from chains of different materials [40].

Three calibrated piezosensors $\left(R C \sim 10^{3} \mu \mathrm{s}\right)$ were connected to a Tektronix oscilloscope to detect force-time curves. Two piezogauges made from lead zirconate titanate (square plates with thickness $0.5 \mathrm{~mm}$ and $3 \mathrm{~mm}$ side) with nickel-plated electrodes and custom microminiature wiring supplied by Piezo Systems, Inc. were embedded inside two of the PTFE and two steel particles in the chains similar to $[8,19,36,37]$. The wired piezoelements were glued between two top parts cut from original beads [Fig. 1(b)]. The calcu- 
lation of the signal speed is based on the time interval between the maxima of the signals detected by the two gauges separated by a known distance (in most cases equal to five particle diameters) simultaneously with the measurement of the force acting inside the particles. The speed of the pulse was related to the averaged force magnitude between the two gauges representing the magnitude of the contact force.

A typical PTFE particle with an embedded sensor consisted of two similar caps (total mass $2 M=0.093 \mathrm{~g}$ ) and a sensor with a mass $m=0.023 \mathrm{~g}$ glued between them. The total mass of these particles was equal to $0.116 \mathrm{~g}$, which is close to the mass of the original PTFE particle $(0.123 \mathrm{~g})$. In theory, the introduction of a particle with a different mass in a chain of particles of equal masses results in weak wave reflections, as observed in $[12,18,20]$. Numerical calculations with a single particle in the chain with a mass $0.116 \mathrm{~g} \mathrm{em}-$ bedded into the chain of particles with mass $0.123 \mathrm{~g}$ created negligible effects of wave reflections [37]. Therefore such small deviation of the sensor mass from the particle mass $(<6 \%)$ in both the PTFE and stainless-steel chains was neglected in the following numerical calculations and, thus, the chains were considered uniform for simplicity.

The third piezogauge, supplied by Kinetic Ceramics, Inc., was bonded with epoxy on two electrode foils for contacts and reinforced by a 1-mm brass plate on the top surface. This sensor was then placed on top of a long vertical steel rod (wave guide) embedded at the other end into a steel block to avoid possible wave reverberation in the system [Fig. 1(a)]. The sensor embedded in the wall was calibrated using the conservation of linear momentum during a single impact by a steel ball as in [37]. Sensors in the particles were calibrated by comparing their signal with the signal from the sensor at the wall under a controlled, relatively long, simultaneous impact loading.

Considering the particles with an embedded sensor as rigid bodies, the forces on the top and bottom of the piezoelectric plate can be easily related to the forces acting on the corresponding contacts of the sensor [37]. This simplification allows a direct comparison of the force-time curves obtained in experiments with the average of the contact forces derived in the numerical calculations. The forces acting on both sides of the sensor deviate symmetrically from the average value of the contact forces if attenuation is negligible. When the measured force reaches its maximum $\left(F_{m, e}\right)$ it is equal to the corresponding forces on the contacts of the particle and on both sides of the piezoelement [37].

To relate the maximum value of the dynamic force measured by the embedded sensor $F_{m, e}$ and the value of the maximum contact force between neighboring particles $F_{m}$, which is present in the equations for solitary wave speed [Eqs. (1)-(3)], we used the coefficient $\beta$ found numerically as explained in the next section:

$$
F_{m}=\beta F_{m, e}+F_{0} .
$$

Strongly nonlinear sonic-vacuum-type materials allow a significant change of speed of propagation using an initial static compressive force, which make them qualitatively different from the linear systems. In this paper, the tunability of the signal propagating through the chains was achieved by adding a magnetically induced preload. In contrast, previous investigations used a preloading of the system through the mechanical application of the load using a wire with attached weights or contact interaction between the last particle and the frame $[4,11]$. The former type of preloading allows application of a constant external force to the end magnetic particle independently from its displacement. This was difficult to achieve in the previous attempts especially if the dynamic displacement of the end particle was comparable to its initial displacement due to the preloading. This case would change boundary conditions in an uncontrollable way. The proposed magnetically induced static precompression ensures fixed boundary conditions which are used in numerical calculations and even allows the additional possibility of a fine-tuning of the preload which can be time dependent. A neodymium-iron-boron ring magnet (internal and external diameters $11.43 \mathrm{~mm}, 53.34 \mathrm{~mm}$, respectively, with mass $242.2 \mathrm{~g} \mathrm{(} \pm 0.1 \mathrm{~g})$ from Dexter Magnetics Technologies) was placed around the PTFE cylinder containing the chain and held in place by the magnetic interaction with the magnetic steel particle placed on the top of the chain [Fig. 1(a)]. Throughout the whole chain the static compressive force induced by the weight of the magnet was $2.38 \mathrm{~N}$ additional to nonuniform gravitational preload. This particular setup was chosen to allow an easy noncontact control over the variation of the static preload by slight axial movements of the magnetic ring or by the use of ring magnets of different masses. A higher level of magnetically induced precompression in experiments $(4.25 \mathrm{~N})$ was achieved by adding mass to the magnet.

Nonmagnetic alumina $\left(\mathrm{Al}_{2} \mathrm{O}_{3}\right)$ cylinders $(0.47 \mathrm{~g}$ and $1.2 \mathrm{~g}$ ) were used in experiments to generate pulses of different durations and force magnitudes in the 1D phononic crystals by controlled impacts on the top particle of the chain. Waves of different magnitudes were excited with different velocities of the striker impacts.

\section{NUMERICAL CALCULATIONS}

For a comparison with experiments, numerical calculations were performed using Matlab. Particles were treated as rigid bodies connected by nonlinear springs according to Hertz law as in previous investigations $[1,2,4,14,19]$. The validity of the Hertzian interaction for a system composed of PTFE beads was extensively studied and demonstrated in [37]. As mentioned earlier, to compare numerical results and experimental data, we calculated an average compression force for solitary waves based on the corresponding compression forces on the particle contacts. The parameters used in numerical calculations to represent the materials properties are listed in Table I.

The coefficient $\beta$ in Eq. (5) is determined numerically as $\beta=F_{d, n} / F_{m, n}$, where $F_{m, n}$ is the maximum value of the force obtained numerically by averaging the dynamic forces on the contacts between adjacent particles and $F_{d, n}$ is the magnitude of these dynamic contact forces. $F_{m, n}$ is analogous to $F_{m, e}$ in experiments as described in [37].

The dependence of the coefficient $\beta$ as a function of the normalized force magnitude in a solitary wave $F_{m, n}$ 
TABLE I. Parameters used in numerical calculations to represent the properties of the materials composing the strongly nonlinear media tested experimentally.

\begin{tabular}{lccc}
\hline \hline & $\begin{array}{c}\text { Elastic modulus } \\
{[\mathrm{GPa}]}\end{array}$ & $\begin{array}{c}\text { Poisson } \\
\text { ratio }\end{array}$ & $\begin{array}{c}\text { Density } \\
{\left[\mathrm{kg} / \mathrm{m}^{3}\right]}\end{array}$ \\
\hline PTFE beads [37,44] & 1.46 & 0.46 & 2200 \\
Stainless-steel beads [45] & 193 & 0.30 & 8000 \\
Red-brass (wall) [46] & 115 & 0.31 & 8750 \\
Alumina (impactor) [47] & 416 & 0.23 & 3900 \\
\hline \hline
\end{tabular}

$\left(F_{m, n} / F_{0}\right)$ is shown in Fig. 2. The same coefficient was introduced in [37] for PTFE-based systems. The coefficient $\beta$ is independent of the elastic modulus, but varies with respect to the force magnitude of the solitary wave approaching one when $F_{m, n}$ is close to zero. This dependence was taken into account when calculating the value of dynamic contact force from measured values by gauges inside particles for both PTFE and stainless-steel cases.

The nonuniform static precompression resulting from the gravitational loading of the particles in the vertical chains was included in calculations and added to the magnetically induced prestress. The numerical calculations included the presence of the magnetic bead at the top of the chain in all cases. Energy and linear momentum (before interaction with a wall) were conserved with a relative error of $10^{-8} \%$ and $10^{-12} \%$ as in [37]. Dissipation was not included in the current numerical analysis.

The force magnitude of the solitary waves was measured with accuracy in the range of $15 \%-30 \%$. The larger error at lower magnitudes is attributed to the higher signal-to-noise ratio. The accuracy of the speed measurements can be estimated within $10 \%$ due to the uncertainty in the sensors alignment (about $1 \mathrm{~mm}$ for each sensor).

\section{RESULTS AND DISCUSSION}

Typical results of the experiments for a chain of 52 PTFE beads capped by the magnetic steel particle are shown in

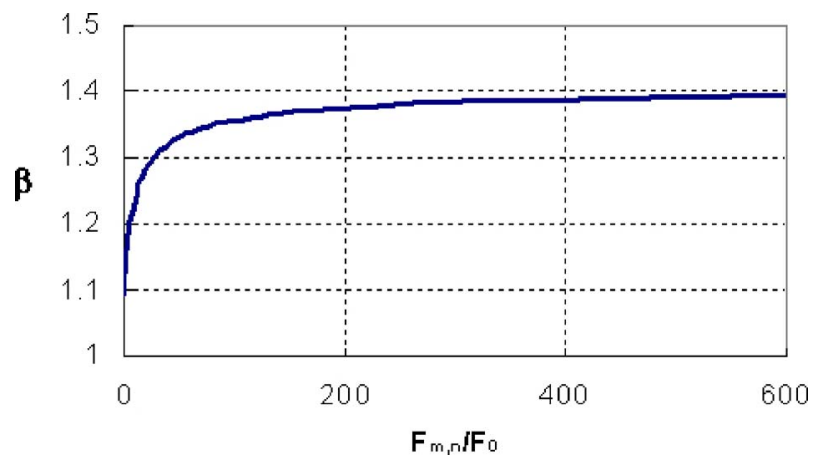

FIG. 2. (Color online) Plot representing the $\beta$ coefficient for solitary waves found in numerical calculations for the stainless-steel case, as a function of the normalized maximum-averaged dynamic force on the contacts $\left(F_{m, n}\right.$ corresponds to $F_{m, e}$ measured by embedded gauges in experiments).
Figs. 3(a) and 3(c). In these experiments, the impulse loading was generated using a 0.47 gram $\mathrm{Al}_{2} \mathrm{O}_{3}$ cylinder with a velocity of $0.89 \mathrm{~m} / \mathrm{s}$ impacting the top of a magnetic particle. Internal sensors were placed in the 20th and 29th beads from the top magnetic particle and the third piezogauge was embedded in the wall. The zero time in experiments corresponding to Figs. 3(a) and 3(c) is arbitrary chosen, and the zero time in numerical calculations [Figs. 3(b) and 3(d)] corresponds to the moment of impact.

The obtained experimental profiles and corresponding numerical results for a gravitationally loaded chain are presented in Figs. 3(a) and 3(b). In this weakly compressed state, the Teflon-based "sonic vacuum" demonstrates the typical fast decomposition of an initial impulse over a distance comparable to the soliton width similar to the trains observed in a shorter 21-particle chain (Fig. 5 in [37]). In Fig. 3(a) the duration of the leading solitary wave pulse was equal to $170 \mu \mathrm{s}$. The speed of its propagation, based on the time interval between the signal's maxima measured by the two embedded sensors, was $129 \mathrm{~m} / \mathrm{s}$. These values result in a solitary wave width equal to 4.6 particles diameters. This agrees reasonably well with the solitary wave width predicted in long-wave theory for a chain of particles interacting according to Hertz' law (five particles diameter) $[1,19]$. These parameters allow one to evaluate the characteristic strain rates for PTFE at the contact area equal to $4 \times 10^{2} \mathrm{~s}^{-1}$ [37]. The experimental results [Figs. 3(a) and $3(\mathrm{c})]$ agreed qualitively with the numerical calculations under the same testing conditions [Figs. 3(b) and 3(d)] as well as in other investigated impact conditions although dissipation is apparent especially at the bottom of the chain (Fig. 3).

Results of numerical calculations demonstrated that magnetically induced static compressive force under a similar type of impact caused a qualitative change of the signal shape from a train of solitary waves in a weakly compressed case [Fig. 3(b)] to a few solitary waves with a periodically oscillating tail [Fig. 3(d)]. In experiments [Fig. 3(c)] the periodic oscillatory tail was not observed, which may be due to the dissipation caused by the interaction between the wall of the holder and the particles as well as dissipation on the contacts.

One of the most interesting consequences of the noncontact magnetically induced static force applied to the chain is a significant change in the speed of the signal propagation. In Figs. 3(a)-3(d) the pulse excited by the same impact resulted in an increased speed from a value of $129 \mathrm{~m} / \mathrm{s}$ for the gravitationally loaded chain to $222 \mathrm{~m} / \mathrm{s}$ for the magnetically preloaded system. This means a $\sim 70 \%$ control over the initial "natural" signal speed of the investigated strongly nonlinear system. The solitary wave speed in only gravitationally loaded PTFE chains is about 2 times smaller than the speed of a solitary wave in the chain of nylon beads [15] with magnitude $1 \mathrm{~N}$ due to a smaller elastic modulus of Teflon in comparison with nylon. This is in agreement with theoretical dependence of speed on elastic modulus [Eq. (1)].

Interestingly, in numerical calculations the dynamic force magnitudes of the incident pulses in the magnetically precompressed chains are close to the one observed in the only gravitationally loaded chains [compare corresponding curves in Figs. 3(b) and 3(d)]. Meanwhile, the dynamic force mag- 

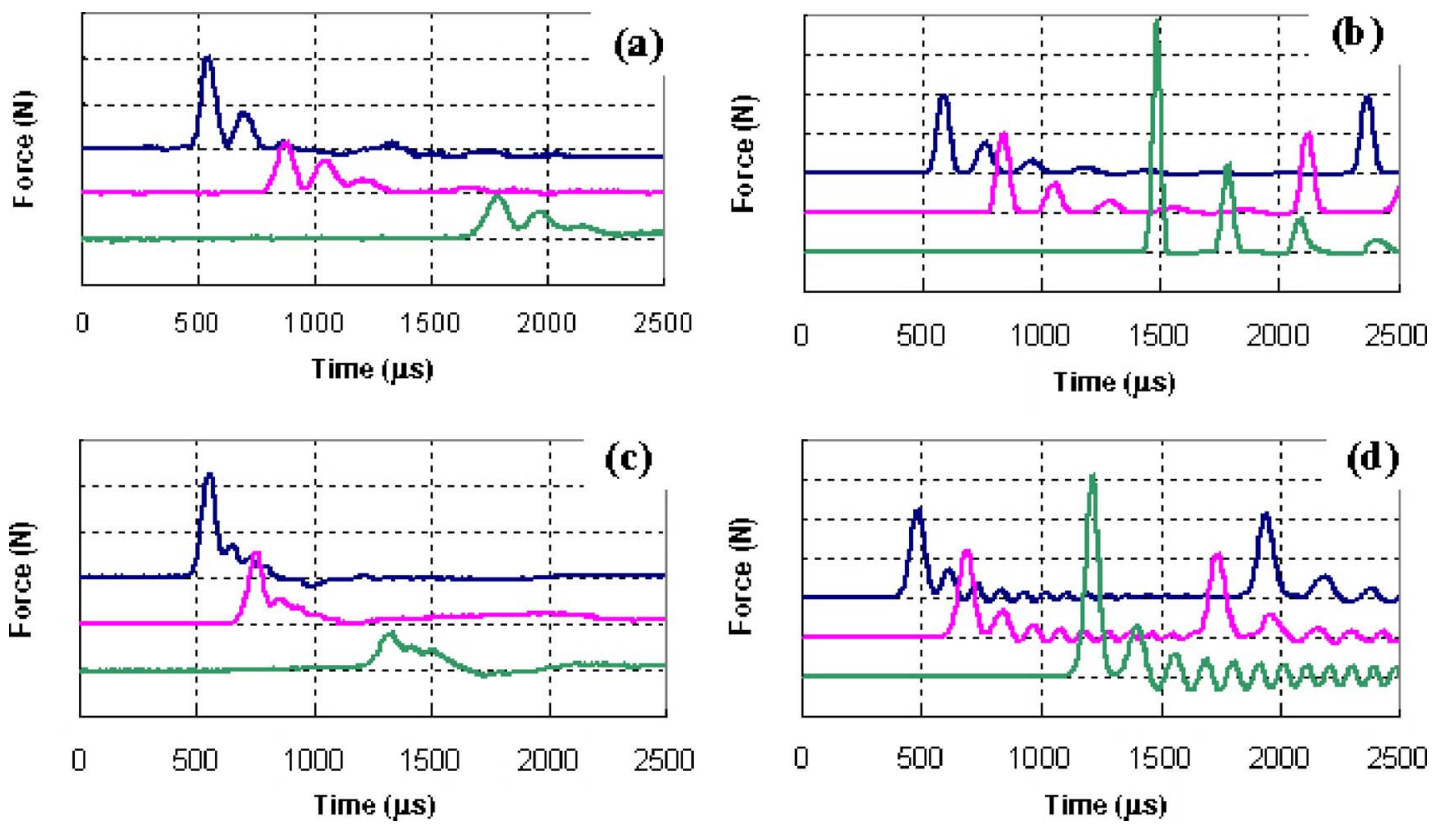

FIG. 3. (Color online) Experimental and numerical results corresponding to a chain of PTFE beads with and without magnetically induced precompression. (a) Forces detected in experiment by the sensor in the 20th ball from the impacted side (top curve), by the sensor in the 29th ball (middle curve), and at the wall (bottom curve) without magnetic precompression; vertical scale is $0.5 \mathrm{~N}$ per division. (b) Numerical calculations of averaged dynamic contact forces between particles and at the wall corresponding to the signals detected by the sensors in (a), without magnetic precompression but including gravitational preload; vertical scale is $2 \mathrm{~N}$ per division. (c) Forces detected in experiment with static magnetically induced precompressive force equal $2.38 \mathrm{~N}$; vertical scale is $1 \mathrm{~N}$ per division. (d) Numerical calculations corresponding to experimental conditions in (c) including forces due to gravitation and magnetic precompression; vertical scale is $2 \mathrm{~N}$ per division.

nitude on the wall in the magnetically precompressed chains in numerical calculations is noticeably smaller than the one in the former case [see bottom curves in Figs. 3(b) and 3(d)]. This shows that the static compressive force can significantly reduce the maximum of the dynamic force acting on the wall though this phenomenon was not observed in the experiments probably due to the decrease of dissipation in the magnetically precompressed chain.

In experiments an impact by the same striker with the same velocity on the gravitationally and magnetically preloaded PTFE chains resulted in an increase of force magnitude by 2 times in the incident pulses in the latter case [compare corresponding curves in Figs. 3(a) and 3(c)]. This is probably due to the significant decrease of dissipation related to the increase of the stiffness and to the reduction of the relative displacement between the particles under similar conditions of loading at higher precompression. The delayed splitting observed in Fig. 3(c) also contributes to the larger amplitude of the leading pulse.

The low-magnitude precursor under magnetic precompression was noticeable especially in the wall gauge [Fig. 3(c), bottom curve]. This may be due to the propagation in front of the leading solitary wave of a high-speed disturbance with the speed of sound in solid PTFE through the central cylindrical column created when the initial precompression flattens the contact area between spherical particles. This phenomenon is not included in the numerical approach and requires further investigation.

Table II shows a summary of the typical results for the magnitude of the dynamic contact forces $F_{d}$ and correspond- ing velocities $V_{s}$ obtained in experiments. A comparison of five representative values obtained experimentally with the corresponding values obtained in numerical calculations and with the theory of long-wave approximation for given values of $F_{d}$ is included for clarity.

The force data presented in the experimental part of the table $\left(F_{d}\right)$ represent the average of the maximum value of the force at the two contact points $\left(F_{d, e}\right)$ extracted from the magnitudes of the corresponding signals of the two embedded gauges with force magnitudes $F_{m, e}$ (where $\left.F_{d, e}=\beta F_{m, e}\right)$. The solitary wave speeds related to $F_{d}$ were obtained by dividing the distance between the sensors (placed in integer intervals of five particle diameters) by the measured peak-to-peak time interval. To calculate the speed of a solitary wave corresponding to the given contact force magnitude $F_{d}$ in the long-wave approximation we used Eq. (1) where the total force $F_{m}$ is the sum of $F_{d}$ plus $F_{0}$ caused by initial precompression (gravitational or gravitational plus magnetic).

The speed of a solitary wave in the long-wave approximation is slightly lower than in the numerical calculations for a gravitationally loaded discrete chain at the same magnitude of dynamic force $F_{d}$ (see left side of Table II). The opposite tendency is characteristic for the case where additional magnetically induced loading is applied (see right side of Table II). In both cases the difference between the longwave approximation and a discrete chain is actually very small, which agrees with the results of [31].

It should be noted that the speeds of the solitary wave at the investigated range of dynamic force magnitudes (which is about 2 times higher than the initial precompression force) 
TABLE II. Comparison of experimental values of the solitary wave speeds $V_{s}$ with the corresponding values obtained in numerical calculation and with the theoretical values from long wave approximation.

\begin{tabular}{|c|c|c|c|c|c|c|c|c|c|c|c|}
\hline \multicolumn{6}{|c|}{ Only gravitational precompression } & \multicolumn{6}{|c|}{ Magnetic and gravitational precompression } \\
\hline \multicolumn{2}{|c|}{ Experimental Data } & \multicolumn{2}{|c|}{$\begin{array}{l}\text { Numerical } \\
\text { results }\end{array}$} & \multicolumn{2}{|c|}{$\begin{array}{l}\text { Long-wave } \\
\text { approximation }\end{array}$} & \multicolumn{2}{|c|}{$\begin{array}{l}\text { Experimental } \\
\text { data }\end{array}$} & \multicolumn{2}{|c|}{$\begin{array}{l}\text { Numerical } \\
\text { results }\end{array}$} & \multicolumn{2}{|c|}{$\begin{array}{c}\text { Long-wave } \\
\text { approximation }\end{array}$} \\
\hline$F_{d}[\mathrm{~N}]$ & $V_{s}[\mathrm{~m} / \mathrm{s}]$ & $F_{d, n}[\mathrm{~N}]$ & $V_{s}[\mathrm{~m} / \mathrm{s}]$ & $F_{d}[\mathrm{~N}]$ & $V_{s}[\mathrm{~m} / \mathrm{s}]$ & $F_{d}[\mathrm{~N}]$ & $V_{s}[\mathrm{~m} / \mathrm{s}]$ & $F_{d, n}[\mathrm{~N}]$ & $V_{s}[\mathrm{~m} / \mathrm{s}]$ & $F_{d}[\mathrm{~N}]$ & $V_{s}[\mathrm{~m} / \mathrm{s}]$ \\
\hline 5.5 & 168 & 5.7 & 184 & 5.7 & 182 & 5.7 & 241 & 5.8 & 228 & 5.8 & 230 \\
\hline 4.3 & 166 & 4.3 & 177 & 4.3 & 174 & 4.5 & 235 & 4.5 & 224 & 4.5 & 227 \\
\hline 3.3 & 167 & 3.3 & 170 & 3.3 & 167 & 3.4 & 230 & 3.2 & 220 & 3.2 & 223 \\
\hline 2.5 & 163 & 2.6 & 163 & 2.6 & 161 & 2.6 & 221 & 2.6 & 218 & 2.5 & 221 \\
\hline 2.1 & 143 & 2.0 & 159 & 2.0 & 155 & 2.0 & 223 & 2.0 & 215 & 2.0 & 220 \\
\hline
\end{tabular}

in the magnetically precompressed chain is slightly higher than the speed of the sound in the system $c_{0}=211.2 \mathrm{~m} / \mathrm{s}$ evaluated from Eq. (3).

The comparison of the shapes of the leading pulses detected experimentally and the stationary solitary waves obtained in separate numerical calculations including a larger number of particles is shown in Fig. 4.

From Fig. 4 we can see that the shape of the leading pulses in experiments is close to the shape of the stationary solitary waves in the numerical calculations despite the fact that in experiments these pulses were not completely separated from the oscillatory tail at the given distances of the sensors from the impacted end of the chain.

In the magnetically precompressed chains, the splitting of the incident pulse into a train of solitary waves is delayed especially when the dynamic force magnitude of the pulse is smaller than or comparable to the initial static compressive force $[4,19,27]$. At a relatively small value of their ratio the strongly nonlinear solitary wave becomes close to the soliton solution of the Korteweg-de Vries wave equation with a width infinitely increasing with dynamic force magnitude decreasing to zero $[4,19]$. Detailed analysis of the depth for solitary wave stabilization depending on the relative magnitude of the solitary wave force and the initial precompressive force can be found in [27]. In the numerical calculations, the leading solitary pulse was formed being completely separated from the oscillatory tail in the vicinity of the 950th particle from the impacted end [curve 2 in Fig. 4(b) corresponds to the 980th particle in the chain included in the numerical calculations]. This may explain why the stationary solitary pulse in numerical calculations is wider than the width of the nonstationary leading pulse in the magnetically precompressed chain experimentally detected at the 20th particle [Fig. 4(b)]. The corresponding increase of the width of the leading pulse when it propagates inside the magnetically precompressed chain was observed in our numerical calculations.

Figure 5 shows the comparison of the experimental results with the theoretical values obtained from the long-wave approximation and the numerical data for discrete chains of both gravitationally (lower curves and experimental points) and magnetically precompressed $(2.38 \mathrm{~N})$ systems (upper curves and corresponding experimental points). It is known that the theoretically predicted speed of solitary waves in strongly nonlinear chains has a strong dependence on the force magnitude represented by Eq. (1) for precompressed chains. The curves based on the long-wave approximation (curves 2 and 4) are very close to the one obtained in numerical calculations (curves 3 and 5). It should be mentioned that this agreement is obtained when only the leading ap-
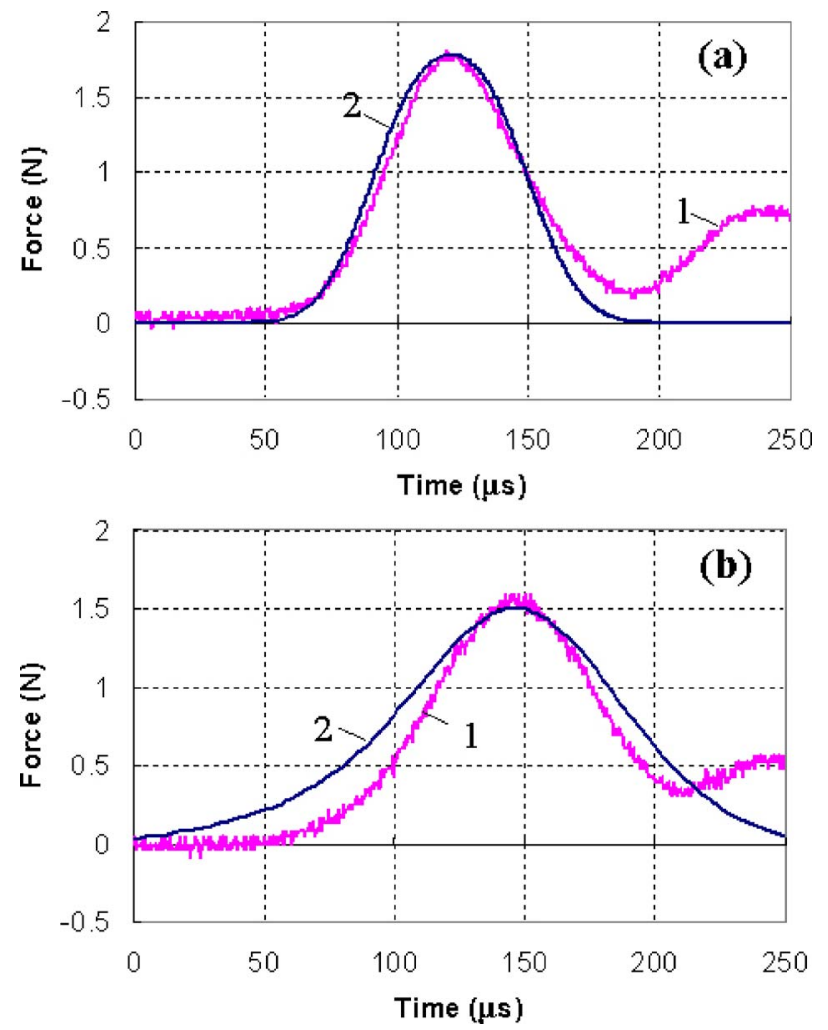

FIG. 4. (Color online) The leading pulses obtained in experiments (curves 1 ) and stationary solitary waves found in numerical calculations (curves 2) with the same force magnitude. (a) PTFE chain under gravitational precompression and the stationary solitary wave calculated in a "sonic vacuum." (b) Magnetically precompressed PTFE chain and the stationary solitary wave calculated in a uniformly precompressed chain with force $2.38 \mathrm{~N}$. In both cases the PTFE chain was composed of 52 particles and a sensor was embedded into the 21 st particle from the top, a magnetic steel particle was positioned on the top, and the impactor was the $0.47 \mathrm{~g} \mathrm{Al}_{2} \mathrm{O}_{3}$ cylinder. 


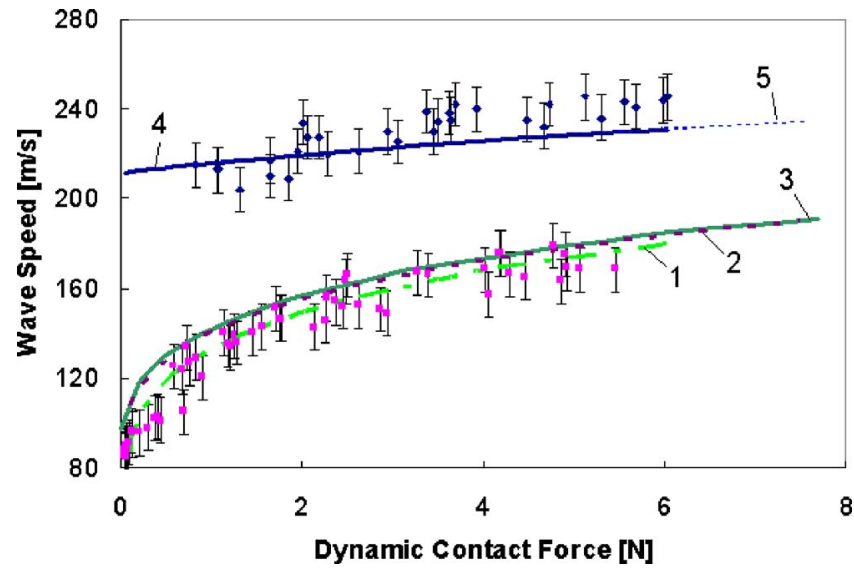

FIG. 5. (Color online) Dependence of the solitary wave speed on the magnitude of the contact dynamic force for gravitationally loaded and for magnetically tuned chains composed of PTFE beads. The experimental values for corresponding curves are shown by solid squares and dots. Curve 1 represents the long-wave approximation for the "sonic vacuum" using an elastic modulus for PTFE equal to $1.46 \mathrm{GPa}$. Curves 2 and 4 are the theoretical curves based on Eq. (1), and the corresponding numerical calculations for the discrete chains are represented by curves 3 and 5 .

proximation was used to connect strains in the continuum limit and forces in discrete chain in Eqs. (1) and (4). Experimental data obtained with an increased precompression (4.25 N, not shown in Fig. 5) also followed the general trend prescribed by the long-wave approximation.

At higher force magnitudes in the solitary wave the agreement between the theoretical data and experiments is better than at lower magnitude for the weakly (gravitationally) precompressed chain. We attribute this behavior to the dependence of the elastic modulus of the dynamically deformed PTFE on strain (and strain rate) on the particle contact [37].

It should be mentioned that the solution obtained with the long-wave approximation [Eq. (1)] for a solitary wave refers only to a uniformly compressed chain with a static force $F_{0}$. The gravitational force, though, results in a nonuniform chain compression. To apply the solution from the long-wave approximation to the gravitationally preloaded and magnetically tuned chains to calculate $V_{s}$, we used a constant value of $F_{0}$ equal to the value of compressive force at the midpoint between the sensors. In the numerical results the local wave speed was calculated taking into account the nonuniform gravitational compression. The small difference observed when comparing the two approaches (compare curves 2-3 and 4-5 in Fig. 5) demonstrated that they are in a very good agreement in this range of static and dynamic forces. Even the "sonic vacuum" approximation [where the gravitational preload is excluded, Eq. (4)] agrees reasonably well with the cases where gravitational compressive forces were taken into account (compare curve 1 with 2 and 3 ) for larger magnitudes of dynamic contact force. At a magnitude of dynamic contact forces close to zero, there is a significant difference between the "sonic vacuum" and the gravitationally precompressed chains. The wave speed in sonic vacuum tends to zero at very low magnitude of dynamic contact forces, while in the gravitationally loaded chains it tends to the value of the corresponding sound speed $c_{0}=92.6 \mathrm{~m} / \mathrm{s}$ with a dynamic elastic modulus for PTFE taken equal to $1.46 \mathrm{GPa}$ [Eq. (2)]. The numerical data also demonstrated the same limit for $c_{0}$. In the low-magnitude range the experimental data are below the long-wave approximation and numerical curves. This may be due to the less than $1.46 \mathrm{GPa}$ dynamic elastic modulus of PTFE at smaller strains (and strain rates) [37].

The increase of the sound speed $c_{0}$ in the PTFE chain under the added magnetically induced precompression $(2.38 \mathrm{~N})$ in comparison with only gravitational preload $\left(0.017 \mathrm{~N}, c_{0}=92.6 \mathrm{~m} / \mathrm{s}\right)$ is about $128 \%$ at practically the same density. This results in a corresponding change of acoustic impedances.

The agreement between the speeds in the magnetically tuned system and the estimated theoretical values means that the equations for the dependence of the solitary wave speed based on the nonlinear continuum approximation can be a reasonable foundation for the design of "sonic-vacuum"-type devices based on PTFE beads. The speed of the solitary wave at the minimum investigated dynamic force magnitude in the magnetically precompressed chain (limit at $F_{\mathrm{d}}$ approaching zero for curves 4 and 5 for long-wave approximation and numerical calculations, respectively) is very close to the speed of the sound in the system $c_{0}=211.2 \mathrm{~m} / \mathrm{s}$ [estimated based on the long-wave approximation, Eq. (2)].

In the experiments with magnetically induced static precompression the maximum total force $(8.4 \mathrm{~N})$ was larger than in the gravitationally precompressed chain. Additionally PTFE was loaded differently in comparison with the weakly compressed chain even when the maximum forces were the same. As mentioned, the elastic modulus of PTFE chains was selected to have a constant value of $1.46 \mathrm{GPa}$ from the extrapolation of the Hugoniot data [44]. Despite a difference in the loading conditions at this large strain the higher value of the elastic modulus (compared to the ultrasonically measured elastic modulus at normal conditions) is able to describe the experimental data. Further research on the value of elastic modulus of PTFE under conditions of dynamic deformation and the application of Hertz' law is necessary to clarify the observed behavior. The dependence of PTFE's elastic modulus on contact strain may cause a deviation of the contact law from a Hertzian-type behavior.

To compare the predictions of the long-wave theory for the tunability of strongly nonlinear systems and relate it with the variation of the elastic modulus of the beads, we measured the parameters of the solitary waves in a stainlesssteel-based system under the same magnetically induced precompression. Stainless-steel particles have more than two orders of magnitude difference in the elastic modulus $(193 \mathrm{GPa})$ with respect to PTFE $(1.46 \mathrm{GPa})$. The experimental results of previous research $[2,11,15,36]$ are in excellent agreement with the predictions of the long-wave theory and with the results of numerical calculations for chains composed of stainless-steel beads. The addition of a prestress in chains composed of steel beads was also examined in [11] but for a range of force magnitudes much higher than the one presented in this study. This paper extends the investigation to multiple solitary waves in contrast to a single solitary wave in [11].

Experimental and numerical results for chains composed of stainless-steel beads are shown in Figs. 6 and 7. Solitary 

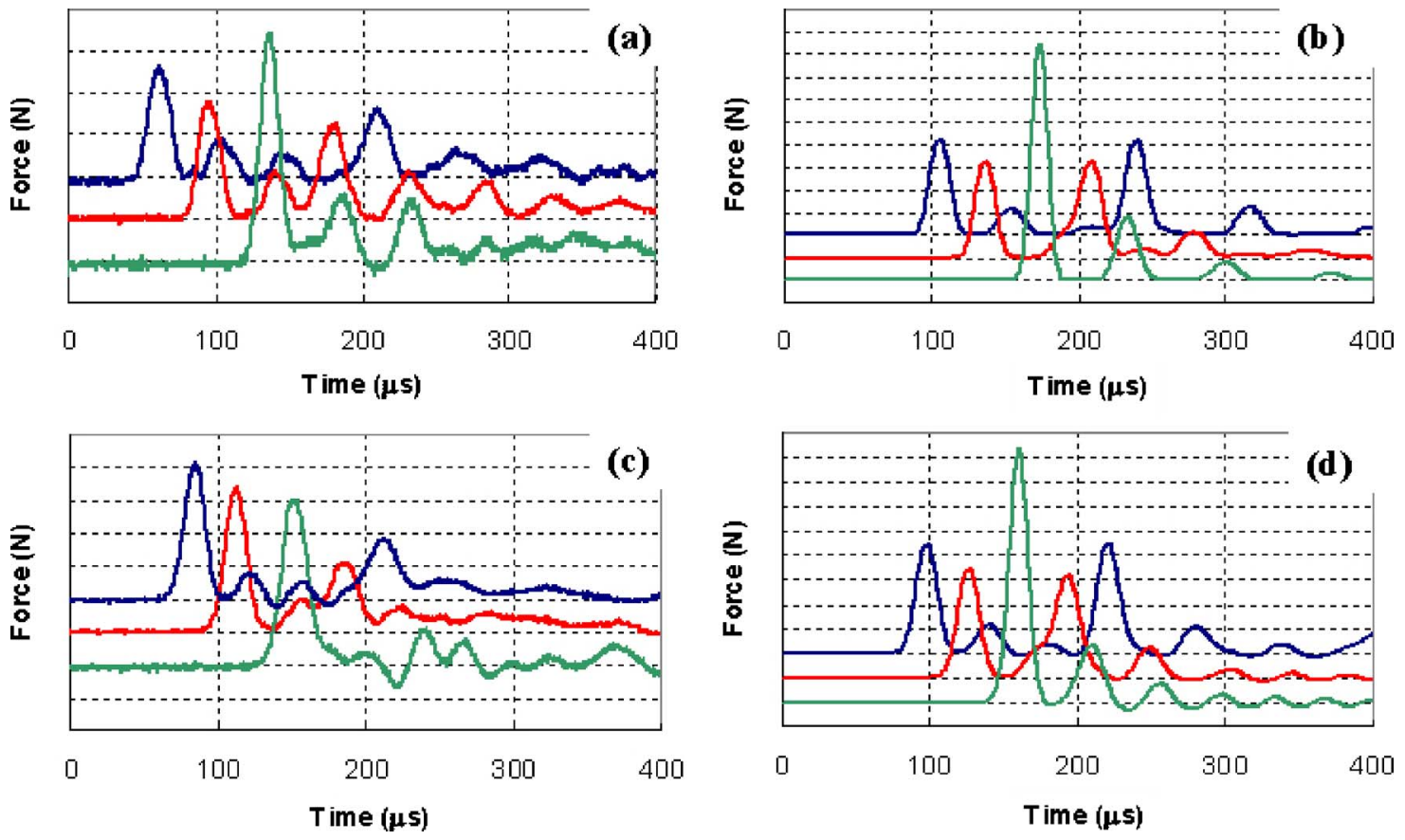

FIG. 6. (Color online) Experimental and numerical results on a chain of 21 stainless-steel (AISI Type 316L) beads with and without magnetic precompression impacted by an alumina striker with a mass equal to $1.2 \mathrm{~g}$ and initial velocity equal to $0.44 \mathrm{~m} / \mathrm{s}$. (a) Force detected in experiment by the sensor in the ninth ball from the wall (top curve), by the sensor in the fifth ball (middle curve) and at the wall (bottom curve) without magnetic precompression. (b) Numerical calculations corresponding to experimental conditions in (a), including gravitational precompression. (c) Force detected in experiment with magnetic precompression equal $2.38 \mathrm{~N}$. (d) Numerical calculations corresponding to experimental conditions in (c), including gravitational and magnetic precompression. Vertical scale is $5 \mathrm{~N}$ per division for all figures (a)-(d).

waves in the chain of stainless-steel beads also demonstrated a significant increase of speed with added magnetically induced prestress of $2.38 \mathrm{~N}$. For example, in experiments the leading solitary pulse presented in Fig. 6(a) had a speed of $580 \mathrm{~m} / \mathrm{s}$ in the gravitationally precompressed chain then increased to $688 \mathrm{~m} / \mathrm{s}$ with the addition of magnetic precompression in Fig. 6(c). This behavior was also observed in the numerical calculations [Figs. 6(b) and 6(d)].

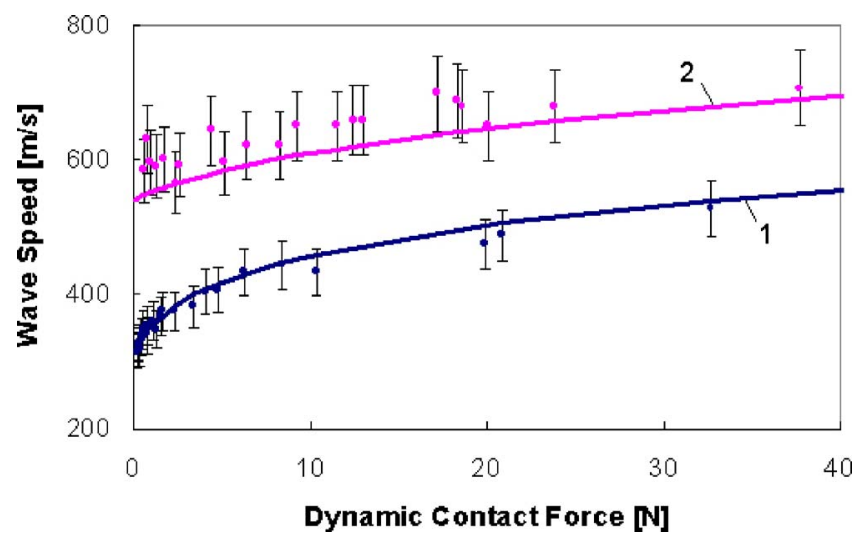

FIG. 7. (Color online) Dependence of the velocity of a solitary wave on the magnitude of the dynamic force for both a gravitationally loaded and magnetically tuned stainless steel chain. Experimental values for corresponding curves are shown by solid dots. Curves 1 and 2 are the theoretical curves based on Eq. (1). The corresponding numerical calculations for discrete chain practically coincide with curves 1 and 2 based on the long-wave approximation.
Similar to the PTFE-based chains, the numerical calculations in the stainless-steel-based system did not show any change of the dynamic force magnitudes of the incident solitary pulses with the application of the initial magnetically induced precompression [see Figs. 6(b) and 6(d)]. This behavior is different than the one observed in experiments, where the added precompression did change the magnitude of the incident pulse [compare Figs. 6(a) and 6(c)]. This difference can be explained by the reduction of dissipation in the precompressed chain in experiments.

It is interesting that a tendency toward a delay of the splitting into a solitary wave train in experiments was observed when the force magnitude of the incident pulse was increased which may be due to the dissipation increase with magnitude of the pulse.

A slight change of the propagating pulse shape was observed in the magnetically precompressed stainless-steel chain. The reduced time interval between the maxima of the incident solitary waves in experiments and in numerical calculations [Figs. 6(c) and 6(d), respectively] demonstrates a significant delay of the pulse splitting. This effect in stainless-steel-based chains is less noticeable than in PTFE [see Figs. 3(c) and 3(d)] which may be due to the smaller speed of signal propagation in the PTFE chains. Also in experiments, in the stainless-steel case, no increase of the wall's pulse magnitude was detected, contrary to the PTFEbased chains. The larger difference between the elastic constant of the wall and that of the PTFE chain can be the cause of this phenomenon.

Figure 7 shows the comparison of the experimental results with the theoretical values for solitary wave speed versus 
dynamic force magnitude obtained from the long-wave approximation [Eq. (1)] of both gravitationally (curve 1 and experimental points) and magnetically (curve 2 and corresponding experimental points) precompressed systems. The curves corresponding to the stainless-steel system extend to a wider range of magnitudes and velocity and the scale is therefore wider than in Fig. 5 for PTFE chains. The curves based on the long-wave approximation and the numerical results coincide for both gravitationally and magnetically precompressed chains. When the dynamic force magnitude approaches zero in the magnetically plus gravitationally precompressed chain $\left(F_{0}=2.44 \mathrm{~N}\right)$, the pulse speed is seen to approach the sound speed $\left(c_{0}=539.6 \mathrm{~m} / \mathrm{s}\right)$ derived from the long-wave approximation [Eq. (2)]. The change of the sound speed $c_{0}$ in the stainless-steel chain under the added magnetically induced precompression $(2.38 \mathrm{~N})$ in comparison with the system under only the gravitational preload $(0.062 \mathrm{~N}$, $\left.c_{0}=292.6 \mathrm{~m} / \mathrm{s}\right)$ is about $84 \%$ at practically the same density, resulting in the corresponding change of acoustic impedances.

These results show that stainless-steel particles under the same static precompressive force demonstrate a higher absolute increase of the solitary wave speed when compared to the PTFE system (compare Figs. 5 and 7) both in experiments and theory and in numerical calculations. Because the PTFE system is elastically much softer, this result can appear counterintuitive. This behavior of the solitary wave speed with static preloading is due to the fact that, in general, a smaller displacement under the same precompression is outweighed by the larger elastic modulus of stainless steel.

These properties of strongly nonlinear phononic crystal can be used for controlled impulse transformation in relatively short transmission lines as well as in tunable acoustic focusing lenses.

\section{CONCLUSIONS}

A method of preloading phononic crystals via magnetic precompression was successfully demonstrated for one- dimensional strongly nonlinear systems based on two qualitatively different materials: elastic (stainless-steel) and viscoelastic (PTFE) beads having more than two orders of magnitude difference in their elastic moduli. This method of precompression ensured well-controlled boundary conditions and the possibility of a time-dependent fine-tuning of the signal speed. The change of the sound speed in these systems under the investigated magnetically induced precompression in comparison with the gravitational preload only is $84 \%$ and $128 \%$ for stainless-steel and PTFE chains, respectively, at practically the same density. They result in the corresponding change of acoustic impedances.

Both systems were investigated for different conditions of loading under the tunable unobtrusive magnetic prestress. A significant tunability of the speed of the signal was achieved over the "natural" uncompressed strongly nonlinear system. The change of the solitary wave speed with prestress in experiments is described very well by the results of the long wave approximation and the numerical data.

The initial preloading significantly reduced dissipation in the experiments in both PTFE and stainless-steel-based systems. A decrease of the reflected wave force magnitude on the wall was observed in numerical calculations for PTFE system. A delay of the splitting of the solitary waves under prestress was observed in experiments and in numerical calculations.

The solitary wave properties obtained in the long-wave approximation are a reliable tool in the designing of sonicvacuum-based devices.

\section{ACKNOWLEDGMENT}

This work was supported by the NSF (Grant No. DCMS03013220).
[1] V. F. Nesterenko, Prikl. Mekh. Tekh. Fiz. 24, 136 (1983) [J. Appl. Mech. Tech. Phys. 24, 733 (1983)].

[2] A. N. Lazaridi and V. F. Nesterenko, Prikl. Mekh. Tekh. Fiz. 26, 115 (1985) [J. Appl. Mech. Tech. Phys. 26, 405 (1985)].

[3] V. F. Nesterenko, Fiz. Goreniya Vzryva 28, 121 (1992).

[4] V. F. Nesterenko, High-Rate Deformation of Heterogeneous Materials (Nauka, Novosibirsk, 1992) (in Russian), Chap. 2.

[5] V. F. Nesterenko, Fiz. Goreniya Vzryva 29, 132 (1993).

[6] S. L. Gavrilyuk and V. F. Nesterenko, Prikl. Mekh. Tekh. Fiz. 34, 45 (1993) [J. Appl. Mech. Tech. Phys. 34, 784 (1993)].

[7] A. Shukla, M. H. Sadd, Y. Xu, and Q. M. Tai, J. Mech. Phys. Solids 41, 1795 (1993).

[8] V. F. Nesterenko, A. N. Lazaridi, and E. B. Sibiryakov, Prikl. Mekh. Tekh. Fiz. 36, 19 (1995) [J. Appl. Mech. Tech. Phys. 36, 166 (1995)].

[9] Y. Zhu, F. Sienkiewicz, A. Shukla, and M. Sadd, J. Eng. Mech. 10, 1050 (1997).
[10] V. F. Nesterenko, J. Phys. IV 4, C8-729 (1994).

[11] C. Coste, E. Falcon, and S. Fauve, Phys. Rev. E 56, 6104 (1997).

[12] M. Manciu, S. Sen, and A. J. Hurd, Physica A 274, 607 (1999).

[13] M. Remoissenet, Waves Called Solitons (Concepts and Experiments), 3rd revised and enlarged ed. (Springer-Verlag, Berlin, 1999).

[14] E. J. Hinch and S. Saint-Jean, Proc. R. Soc. London, Ser. A 455, 3201 (1999).

[15] C. Coste and B. Gilles, Eur. Phys. J. B 7, 155 (1999).

[16] J. Hong, J.-Y. Ji, and H. Kim, Phys. Rev. Lett. 82, 3058 (1999).

[17] A. Chatterjee, Phys. Rev. E 59, 5912 (1999).

[18] E. Hascoet and H. J. Herrmann, Eur. Phys. J. B 14, 183 (2000).

[19] V. F. Nesterenko, Dynamics of Heterogeneous Materials 
(Springer-Verlag, New York, 2001), Chap. 1.

[20] S. Sen, M. Manciu, R. S. Sinkovits, and A. J. Hurd, Granular Matter 3, 33 (2001).

[21] M. Manciu, S. Sen, and A. J. Hurd, Physica D 157, 226 (2001).

[22] M. Manciu, S. Sen, and A. J. Hurd, Phys. Rev. E 63, 016614 (2001).

[23] J. B. Hong and A. G. Xu, Phys. Rev. E 63, 061310 (2001).

[24] S. Sen and M. Manciu, Phys. Rev. E 64, 056605 (2001).

[25] F. S. Manciu and S. Sen, Phys. Rev. E 66, 016616 (2002).

[26] J. Hong and A. Xu, Appl. Phys. Lett. 81, 4868 (2002).

[27] C. A. Arancibia-Bulnes and J. C. Ruiz-Suarez, Physica D 168, 159 (2002).

[28] A. Rosas and K. Lindenberg, Phys. Rev. E 68, 041304 (2003).

[29] R. Reigada, A. Samiento, and K. Lindenberg, Chaos 13, 646 (2003).

[30] C. Daraio, V. F. Nesterenko, and S. Jin, in Shock Compression of Condensed Matter-2003, edited by M. D. Furnish, Y. M. Gupta, and J. W. Forbes, AIP Conf. Proc. No. 706 (AIP, Melville, NY, 2004), pp. 197-200.

[31] A. Rosas and K. Lindenberg, Phys. Rev. E 69, 037601 (2004).

[32] A. I. Musienko and L. I. Manevich, Phys. Usp. 47, 797 (2004).

[33] M. Kastner, Phys. Rev. Lett. 92, 104301 (2004).

[34] M. Kastner, Nonlinearity 17, 1923 (2004).
[35] J. Hong, Phys. Rev. Lett. 94, 108001 (2005).

[36] S. Job, F. Melo, S. Sen, and A. Sokolow, Phys. Rev. Lett. 94, 017802 (2005).

[37] C. Daraio, V. F. Nesterenko, E. Herbold, and S. Jin, Phys. Rev. E 72, 016603 (2005).

[38] L. Vergara, Phys. Rev. Lett. 94, 108002 (2005).

[39] R. L. Doney and S. Sen, Phys. Rev. E 72, 041304 (2005).

[40] V. F. Nesterenko, C. Daraio, E. B. Herbold, and S. Jin, Phys. Rev. Lett. 95, 158702 (2005).

[41] F. J. Zerilli and R. W. Armstrong, in Shock Compression of Condensed Matter, edited by M. D. Furnish, N. N. Thadhani, and Y. Horie (AIP, AIP Conf. Proc. 620, 2002), pp. 657-660.

[42] G. W. Ehrenstein, Polymeric Materials: Structure, Properties, Applications (Hanser, Munich, 2001), p. 256.

[43] DuPont Product Information, Comparison of Different DuPont Fluoropolymers, www.dupont.com/teflon/chemical/.

[44] W. J. Carter and S. P. Marsh, Hugoniot Equation of State of Polymers, Los Almos National Laboratory Preprint, LA13006-MS (1995).

[45] ASM Metals Reference Book, 2nd ed. (ASM, Metals Park, OH, 1983), 2nd. ed., p. 268.

[46] AISI Type 316L, Mechanical Properties, http:// www.efunda.com.

[47] R. G. Munro, J. Am. Ceram. Soc. 80, 1919 (1997). 